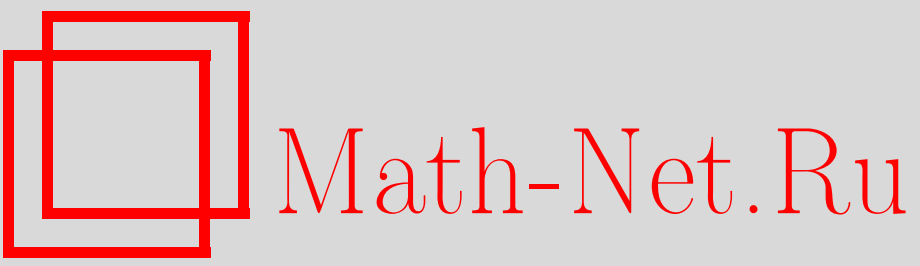

М. В. Горбатенко, Принцип наименьшего действия в общей теории относительности, ТМФ, 1998, том 115, номер 2, 305-311

DOI: https://doi.org/10.4213/tmf875

Использование Общероссийского математического портала Math-Net.Ru подразумевает, что вы прочитали и согласны с пользовательским соглашением

http://www.mathnet.ru/rus/agreement

Параметры загрузки:

IP: 54.174 .149 .18

26 апреля 2023 г., 02:16:43 
ТЕОРЕТИЧЕСКАЯ

И МАТЕМАТИЧЕСКАЯ

ФИЗИКА

Том 115, № 2

май, 1998

(C) $\mathbf{1 9 9 8}$ г.

\section{ПРИНЦИП НАИМЕНЬШЕГО ДЕЙСТВИЯ В ОБЩЕЙ ТЕОРИИ ОТНОСИТЕЛЬНОСТИ}

В рамках общей теории относительности модифицируется процедура получения уравнений Эйнштейна путем введения в римановом пространстве конгруэнции времениподобных кривых и использования связей между вариациями. Цель модификации обеспечить выполнение принципа наименьшего действия по отношению к любой системе отсчета.

\section{1. ВВЕДЕНИЕ}

В обычной формулировке принципа наименьшего действия (ПНД) в обшей теории относительности вторая вариация действия

$$
I=-\int_{\Omega} d \Omega \sqrt{-g} R
$$

не является, вообше говоря, знакоопределенной в окрестности экстремалей. При обсуждении этого вопроса в книге [1, с. 348$]$ отмечается, что:

1) “... можно наложить на $g_{\alpha \beta}$ такие дополнительные условия, при соблюдении которых действие имеет минимум по отношению к варьированию $g_{\alpha \beta}$ ", кроме того, необходимо “... потребовать выполнения этих условий при варьировании";

2) “... все сказанное не влияет на вывод уравнений поля из принципа наименьшего действия."

В качестве четырех дополнительных условий в [1] используются условия

$$
\delta g_{0 k}=0, \quad \delta \operatorname{det}\left(g_{m n}\right)=0 .
$$

В данной работе предложен более последовательный, чем в [1], учет в ПНД идей, содержашихся в приведенных выше двух утверждениях. Бо́льшая последовательность означает следующее:

Во-первых, для обеспечения независимости факта минимальности действия на экстремали от выбора системы отсчета мы с самого начала вводим в теорию конгруэнцию

* Российский федеральный ядерный центр, Всероссийский НИИ экспериментальной физики, г. Саров, Нижегородская обл., Россия. E-mail: 0722@spd.vniief.ru 
времениподобных кривых и ограничения на вариации (2) формулируем в ковариантной форме с помощью векторов 4-скорости $u^{\alpha}(x)$, касательных к кривым конгруэнции.

Во-вторых, используется хорошо известная процедура получения динамических уравнений при наличии связей, в которой связи вводятся в вариацию действия с помощью неопределенных множителей Лагранжа.

Несколько слов о смысле этих модификаций ПНД, детальное изложение которых является предметом данной работы. Первую модификацию мы вынуждены сделать по той причине, что в противном случае знак константы гравитационного взаимодействия в одной системе отсчета будет положительным, а в другой - отрицательным. Если же минимальность действия обеспечивается ковариантным образом (именно для этой цели вводится конгруэнция кривых), то знак константы гравитационного взаимодействия во всех системах отсчета один и тот же. Вторая модификация вопреки приведенному выше мнению [1] является операцией, далеко не безобидной с точки зрения влияния на динамические уравнения. Она приводит к тому, что либо в теории необходимо ограничиваться шестью "рабочими” степенями свободы, построенными из компонент метрики, либо в теории появляется ненулевой тензор энергии-импульса $T_{\alpha \beta}$, построенный из множителей Лагранжа и 4-скорости.

\section{2. ВАРИАЦИЯ ДЕЙСТВИЯ}

Выражение для вариации действия находим по формуле

$$
\delta I=-\int_{\Omega} d \Omega(\sqrt{-g}+\delta \sqrt{-g})\left(g^{\alpha \beta}+\delta g^{\alpha \beta}\right)\left(R_{\alpha \beta}+\delta R_{\alpha \beta}\right)+\int_{\Omega} d \Omega \sqrt{-g} R .
$$

В качестве основной величины, через которую будем выражать все другие вариации, примем $\delta g_{\alpha \beta}$. Выражения для вариаций величин, входящих в $(3)$, имеют следующий вид:

$$
\begin{aligned}
& \left\{\begin{aligned}
\delta\left(\sqrt{-g} g^{\alpha \beta}\right) & =\delta_{1}\left(\sqrt{-g} g^{\alpha \beta}\right)+\delta_{2}\left(\sqrt{-g} g^{\alpha \beta}\right)+\cdots \\
\delta_{1}\left(\sqrt{-g} g^{\alpha \beta}\right) & =\sqrt{-g}\left\{-g^{\alpha \mu} g^{\beta \nu}+\frac{1}{2} g^{\alpha \beta} g^{\mu \nu}\right\} \delta g_{\mu \nu} \\
\delta_{2}\left(\sqrt{-g} g^{\alpha \beta}\right)= & \sqrt{-g}\left\{g^{\alpha \mu} g^{\nu \tau} g^{\rho \beta}-\frac{1}{2} g^{\alpha \mu} g^{\beta \nu} g^{\tau \rho}-\right. \\
& \left.-\frac{1}{4} g^{\alpha \beta} g^{\mu \tau} g^{\nu \rho}+\frac{1}{8} g^{\alpha \beta} g^{\mu \nu} g^{\tau \rho}\right\}\left(\delta g_{\mu \nu}\right)\left(\delta g_{\tau \rho}\right)
\end{aligned}\right. \\
& \delta R_{\alpha \beta}=\left[\delta \Gamma^{\kappa}{ }_{\alpha \beta}\right]_{; \kappa}-\left[\delta \Gamma^{\sigma}{ }_{\alpha \sigma}\right]_{; \beta}+\left(\delta \Gamma^{\kappa}{ }_{\alpha \beta}\right)\left(\delta \Gamma^{\sigma}{ }_{\kappa \sigma}\right)-\left(\delta \Gamma^{\kappa}{ }_{\alpha \sigma}\right)\left(\delta \Gamma^{\sigma}{ }_{\beta \kappa}\right) ; \\
& \left\{\begin{aligned}
\delta \Gamma_{\alpha \beta}^{\lambda}=\delta_{1} \Gamma_{\alpha \beta}^{\lambda}+\delta_{2} \Gamma_{\alpha \beta}^{\lambda}+\cdots \\
\delta_{1} \Gamma_{\alpha \beta}^{\lambda}=\frac{1}{2} g^{\lambda \kappa}\left\{\left(\delta g_{\kappa \beta}\right)_{; \alpha}+\left(\delta g_{\alpha \kappa}\right)_{; \beta}-\left(\delta g_{\alpha \beta}\right)_{; \kappa}\right\} \\
\delta_{2} \Gamma_{\alpha \beta}^{\lambda}=\frac{1}{2}\left(\delta_{1} g^{\lambda \kappa}\right)\left\{\left(\delta g_{\kappa \beta}\right)_{; \alpha}+\left(\delta g_{\alpha \kappa}\right)_{; \beta}-\left(\delta g_{\alpha \beta}\right)_{; \kappa}\right\}
\end{aligned}\right.
\end{aligned}
$$


После подстановки (4)-(6) в (3) выражение для $\delta I$ может быть представлено в виде суммы четырех членов:

$$
\delta I=\delta_{1} I+\delta_{2} I+\delta_{3} I+\delta_{4} I
$$

где

$$
\begin{gathered}
\delta_{1} I=-\int_{S(\Omega)} d S_{\kappa}\left\{\delta \Gamma^{\kappa}{ }_{\alpha \beta}\left[\sqrt{-g} g^{\alpha \beta}+\delta\left(\sqrt{-g} g^{\alpha \beta}\right)\right]-\right. \\
\left.-\left[\sqrt{-g} g^{\kappa \alpha}+\delta\left(\sqrt{-g} g^{\kappa \alpha}\right)\right] \delta \Gamma^{\sigma}{ }_{\alpha \sigma}\right\}, \\
\delta_{2} I=-\int_{\Omega} d \Omega \delta\left(\sqrt{-g} g^{\alpha \beta}\right) R_{\alpha \beta}, \\
\delta_{3} I=\int_{\Omega} d \Omega\left\{\left(\delta\left(\sqrt{-g} g^{\alpha \beta}\right)\right)_{; \kappa} \delta \Gamma^{\kappa}{ }_{\alpha \beta}-\left(\delta\left(\sqrt{-g} g^{\alpha \beta}\right)\right)_{; \beta} \delta \Gamma_{\alpha \sigma}^{\sigma}\right\}, \\
\delta_{4} I=-\int_{\Omega} d \Omega \sqrt{-g} g^{\alpha \beta}\left\{\left(\delta \Gamma^{\kappa}{ }_{\alpha \beta}\right)\left(\delta \Gamma^{\sigma}{ }_{\kappa \sigma}\right)-\left(\delta \Gamma^{\kappa}{ }_{\alpha \sigma}\right)\left(\delta \Gamma_{\beta \kappa}^{\sigma}\right)\right\} .
\end{gathered}
$$

Слагаемое $\delta_{1} I$ является интегралом по гиперповерхности, охватьвающей 4-объем интегрирования. Остальные три слагаемых - интегралы по 4-объему.

\section{3. СВЯЗИ. ДИНАМИЧЕСКИЕ УРАВНЕНИЯ}

Запишем вариацию $\delta_{1} I$ в соотношении (7) следующим образом:

$$
\begin{aligned}
\delta_{1} I= & -\int_{S(\Omega)} d S_{\kappa} \sqrt{-g}\left[g^{\alpha \beta}\left(\delta_{1} \Gamma^{\kappa}{ }_{\alpha \beta}\right)-g^{\kappa \lambda}\left(\delta_{1} \Gamma_{\lambda \sigma}^{\sigma}\right)\right]- \\
& -\int_{\Omega} d \Omega \sqrt{-g}\left(\delta_{1} g^{\alpha \beta}\right)\left\{R_{\alpha \beta}-\frac{1}{2} g_{\alpha \beta} R\right\} .
\end{aligned}
$$

В окрестности экстремали величина $\delta_{1} I$ равна нулю относительно любых вариаций $\delta g_{\alpha \beta}$ внутри 4-объема $\Omega$ при условии обрашения $\delta g_{\alpha \beta}$ в нуль на границе $S(\Omega)$. Вместе с $\delta g_{\alpha \beta}$ на границе обращаются в нуль и все производные от $\delta g_{\alpha \beta}$ по пространственным направлениям (но не по времени).

Запишем поверхностный интеграл из (12) в виде

$$
\int_{S(\Omega)} d S_{\kappa} \sqrt{-g} Y^{\kappa}, \quad Y^{\kappa} \equiv g^{\alpha \beta}\left(\delta_{1} \Gamma^{\kappa}{ }_{\alpha \beta}\right)-g^{\kappa \lambda}\left(\delta_{1} \Gamma_{\lambda \sigma}^{\sigma}\right)
$$

и сделаем два важных предположения. Во-первых, предположим, что вариационный принцип может быть сформулирован для риманова пространства только при дополнительном оснащении этого пространства конгруэнцией времениподобных мировых линий с полем единичных касательных векторов $u^{\alpha}(x)$. Во-вторых, мы предполагаем, что вариационный принцип должен формулироваться для фиксированной в процессе варьирования системы отсчета, связанной с введенной конгруэнцией времениподобных мировых 
линий. Условиями, фиксируюшими во всему 4-объему $\Omega$ систему отсчета, будем считать следующие:

$$
P^{\mu \nu}\left(\delta P_{\mu \nu}\right)=0, \quad u^{\alpha}\left(\delta g_{\alpha \beta}\right) P^{\beta \lambda}=0 .
$$

Здесь $P_{\alpha \beta}=g_{\alpha \beta}-u_{\alpha} u_{\beta}$-метрика пространства, ортогонального к $u^{\alpha}$. Условия (14) являются ковариантным случаем условий (2). Ковариантность обеспечивается тем, что, во-первых, условия формулируются в терминах не компонент метрики (как в [1]), а их вариаций, являющихся полноценными тензорами; во-вторых, для выделения вариаций, подчиняюшихся связям, используется не какая-то случайная система (как в [1]), а система отсчета, связанная с векторным полем $u^{\alpha}(x)$.

Как только в теории возникают связи для класса допустимых вариаций, сразу появляется необходимость в модификации процедуры получения динамических уравнений [2]. Мы должны либо производить варьирование только по тем компонентам метрики, которые не ограничиваются связями, либо пользоваться методом неопределенных множителей Лагранжа. Применительно к "рабочим" степеням свободы метрики оба метода естественно приводят к одним и тем же уравнениям. Однако метод неопределенных множителей дает более полную информацию о системе. Он позволяет получить уравнения не только для "рабочих" степеней свободы метрики, но и для полей, соответствуюших "реакциям опоры", обеспечиваюшим выполнение ПНД. По этой причине в данной работе используется метод неопределенных множителей Лагранжа (для альтернативного способа получения уравнений мы приведем лишь вид получающихся уравнений). В соответствии с этим методом связи (14) с помощью множителей Лагранжа $\varphi, \Phi_{\lambda}$ вводим в объемньй интеграл:

$$
\begin{aligned}
\delta_{1} I= & -\int_{S(\Omega)} d S_{\kappa} \sqrt{-g} Y^{\kappa}-\int_{\Omega} d \Omega \sqrt{-g}\left(\delta_{1} g^{\alpha \beta}\right) \times \\
& \times\left\{R_{\alpha \beta}-\frac{1}{2} g_{\alpha \beta} R-\varphi P_{\alpha \beta}-\frac{1}{2}\left[u_{\alpha} \Phi_{\beta}+u_{\beta} \Phi_{\alpha}\right]\right\} .
\end{aligned}
$$

При написании (15) учтено, что по способу своего введения вектор $\Phi_{\lambda}$ ортогонален вектору $u^{\alpha}$, т.е. $u^{\nu} \Phi_{\nu}=0$. Отсюда следует, что из четырех компонент вектора $\Phi_{\lambda}$ независимы только три.

Представим вариацию $\delta g_{\alpha \beta}$ в следуюшем виде:

$$
\begin{aligned}
\delta g_{\alpha \beta}= & u_{\alpha} u_{\beta} u^{\mu} u^{\nu} \delta g_{\mu \nu}+\left[P_{\alpha}{ }^{\mu} P_{\beta}{ }^{\nu}-\frac{1}{3} P_{\alpha \beta} P^{\mu \nu}\right] \delta g_{\mu \nu}+ \\
& +\left[u_{\alpha} P_{\beta}{ }^{\mu} u^{\nu}+u_{\beta} P_{\alpha}{ }^{\mu} u^{\nu}\right] \delta g_{\mu \nu}+\frac{1}{3} P_{\alpha \beta} P^{\mu \nu} \delta g_{\mu \nu} .
\end{aligned}
$$

В верхней строчке соотношения (16) стоят 6 независимых вариаций, а в нижней 4 вариации, равные нулю в силу связей (14). При независимых вариациях выражение в фигурных скобках в (15) должно быть равно нулю в силу динамических уравнений. При 
нулевых вариациях множители Лагранжа подбираются так, чтобы выражение в фигурных скобках в (15) также было равно нулю. В результате из (15) следуют динамические полевые уравнения в форме уравнений Эйнштейна с ненулевой правой частью

$$
\begin{gathered}
R_{\alpha \beta}-\frac{1}{2} g_{\alpha \beta} R=T_{\alpha \beta}, \\
T_{\alpha \beta}=\left[g_{\alpha \beta}-u_{\alpha} u_{\beta}\right] \varphi+\frac{1}{2}\left[u_{\alpha} \Phi_{\beta}+u_{\beta} \Phi_{\alpha}\right] .
\end{gathered}
$$

Обрашение в нуль объемного интеграла в выражении (15) еше не означает автоматического обрашения в нуль величины $\delta_{1} I$. Однако нетрудно заметить, что в $Y^{\kappa}$ входят производные по времени только от тех вариаций, которые тождественно равны нулю в силу связей (14). Поэтому при нашем способе варьирования поверхностный интеграл, содержашийся в $\delta_{1} I$, равен нулю.

Векторное поле $u^{\alpha}(x)$, фигурируюшее в тензоре $T_{\alpha \beta}$, имеет чисто кинематический характер. Это следует, например, из того обстоятельства, что уравнения (17) могут быть записаны в синхронной по вектору $u^{\alpha}(x)$ системе координат. В этой системе по определению вектор имеет компоненты $u^{\alpha}=(1,0,0,0)$. С другой стороны, присутствие в теории конгруэнции времениподобных мировых линий гарантирует принципиальную возможность исследования уравнений (17) в конечной пространственно-временной 4-мерной области (более подробно о монадном формализме, основанном на конгруэнции времениподобных мировых линий, см., например, в [3]).

В заключение приведем также модифицированные уравнения Эйнштейна, которые получаются при альтернативном способе учета ограничений (14). При альтернативном способе множители Лагранжа вообще не появляются в схеме, а искомыми функциями являются функции

$$
u^{\alpha} u^{\beta} g_{\alpha \beta}, \quad\left[P_{\alpha}^{\mu} P_{\beta}^{\nu}-\frac{1}{3} P_{\alpha \beta} P^{\mu \nu}\right] g_{\mu \nu} .
$$

Для этих функций модифицированные уравнения записываются в следуюшем виде:

$$
\begin{gathered}
u^{\alpha} u^{\beta}\left(R_{\alpha \beta}-\frac{1}{2} g_{\alpha \beta} R\right)=0, \\
{\left[P_{\alpha}^{\mu} P_{\beta}^{\nu}-\frac{1}{3} P_{\alpha \beta} P^{\mu \nu}\right]\left(R_{\mu \nu}-\frac{1}{2} g_{\mu \nu} R\right)=0 .}
\end{gathered}
$$

\section{4. ЗНАКООПРЕДЕЛЕННОСТЬ ВТОРОЙ ВАРИАЦИИ ДЕЙСТВИЯ}

Во втором порядке по $\delta g_{\alpha \beta}$ в слагаемом $\delta_{1} I$ все члены содержат произведение производной от вариации на саму вариацию. Поскольку на границе 4-объема вариации должны обрашаться в нуль, то отсюда следует, что слагаемое $\delta_{1} I$, представляющее собой поверхностный интеграл, не дает вклада во вторую вариацию действия.

Для выяснения вопроса, имеем ли мы дело с минимумом, максимумом или просто с экстремумом действия, необходимо проводить анализ тех членов в слагаемых $\delta_{2} I, \delta_{3} I$, $\delta_{4} I$, которые содержат производные от вариаций по времени. Таких членов заведомо 
нет в слагаемом $\delta_{2} I$. Поэтому мы его в дальнейшем детально рассматривать не будем. В слагаемых $\delta_{3} I$ и $\delta_{4} I$ такие члены имеются. Приведем их в явной форме:

$$
\begin{aligned}
\tilde{\delta} I \equiv & \delta_{3} I+\delta_{4} I \Rightarrow \frac{1}{4} \int_{\Omega} d \Omega \sqrt{-g} g^{\alpha \beta} g^{\mu \nu} g^{\kappa \lambda}\left\{-\left(\delta g_{\alpha \beta}\right)_{; \kappa}\left(\delta g_{\mu \nu}\right)_{; \lambda}+\right. \\
& \left.+2\left(\delta g_{\alpha \beta}\right)_{; \kappa}\left(\delta g_{\lambda \mu}\right)_{; \nu}+\left(\delta g_{\alpha \mu}\right)_{; \kappa}\left(\delta g_{\beta \nu}\right)_{; \lambda}-2\left(\delta g_{\alpha \mu}\right)_{; \kappa}\left(\delta g_{\beta \lambda}\right)_{; \nu}\right\} .
\end{aligned}
$$

Знак $\Rightarrow$ в (19) означает, что в выражении для $\tilde{\delta} I$ мы опустили все члены, в которых нет производных от вариации метрики.

Запишем теперь (19) в синхронной системе координат, в которой ось времени совпадает с направлением вектора $u^{\alpha}(x)$. Подынтегральное выражение в $\tilde{\delta} I$ принимает тогда вид

$$
\begin{aligned}
\tilde{\delta} I \Rightarrow & \frac{1}{4}\left\{2\left(\delta g_{00}\right)_{\mid k}\left(\delta g_{0 k}\right)_{\mid 0}-2\left(\delta g_{00}\right)_{\mid 0}\left(\delta g_{0 k}\right)_{\mid k}+2\left(\delta g_{0 l}\right)_{\mid 0}\left(\delta g_{k k}\right)_{\mid l}-\right. \\
& -2\left(\delta g_{00}\right)_{\mid l}\left(\delta g_{k k}\right)_{\mid l}-2\left(\delta g_{0 l}\right)_{\mid k}\left(\delta g_{0 k}\right)_{\mid l}+2\left(\delta g_{0 k}\right)_{\mid l}\left(\delta g_{0 k}\right)_{\mid l}+ \\
& +2\left(\delta g_{0 l}\right)_{\mid l}\left(\delta g_{k k}\right)_{\mid 0}-\left(\delta g_{l l}\right)_{\mid 0}\left(\delta g_{k k}\right)_{\mid 0}-4\left(\delta g_{0 l}\right)_{\mid k}\left(\delta g_{l k}\right)_{\mid 0}+ \\
& +\left(\delta g_{k l}\right)_{\mid 0}\left(\delta g_{k l}\right)_{\mid 0}+2\left(\delta g_{k l}\right)_{\mid l}\left(\delta g_{00}\right)_{\mid k}-2\left(\delta g_{m l}\right)_{\mid l}\left(\delta g_{k k}\right)_{\mid m}+ \\
& \left.+\left(\delta g_{l l}\right)_{\mid m}\left(\delta g_{k k}\right)_{\mid m}+2\left(\delta g_{m k}\right)_{\mid l}\left(\delta g_{m l}\right)_{\mid k}-\left(\delta g_{k l}\right)_{\mid m}\left(\delta g_{k l}\right)_{\mid m}\right\}
\end{aligned}
$$

В (20) по дважды повторяющимся латинским индексам подразумевается суммирование от 1 до 3 .

После отбрасывания в (20) членов, обрашаюшихся в нуль в силу связей (14), оказывается, что остаются только члены с квадратами пространственных производных и квадратами временных производных. Последние и определяют знак $\tilde{\delta} I$, поскольку достаточно быстрым изменением малых вариаций во времени величину $\tilde{\delta} I$ можно сделать сколь угодно большой. Оставляя только определяюшие члены, получаем, что

$$
\tilde{\delta} I \Rightarrow \frac{1}{4}\left(\delta g_{m n}\right)_{\mid 0}\left(\delta g_{m n}\right)_{\mid 0}>0 .
$$

Это означает, что вариационный принцип в той форме, в которой мы его используем, обеспечивает не экстремальность, а минимальность действия. Естественно, этот результат имеет ковариантный характер, поскольку величина (19) является скаляром, а синхронная система понадобилась лишь для нахождения характера зависимости этой скалярной функции от вариаций метрики.

\section{5. ЗАКЛЮЧЕНИЕ}

Уравнения (17) с отличным от нуля тензором $T_{\alpha \beta}$ в правой части представляют собой модифицированные уравнения Эйнштейна. С первого взгляда ничего особенного с уравнениями не произошло, потому что тензор $T_{\alpha \beta}$ аналогичен феноменологическому тензору энергии-импульса $T_{\alpha \beta}^{\text {matter }}$, включение которого в правую часть уравнений 
является обычным отправным пунктом большинства исследований по общей теории относительности. Однако, если рассматривать тензор (17) как тензор энергии-импульса какой-то материи, то эта материя будет обладать очень специфическим свойством. Она имеет давление, но в системе отсчета, сопутствуюшей вектору $u^{\alpha}(x)$, у нее нет плотности энергии. Обычная материя с таким уравнением состояния не сушествует. Возможная трактовка величины $\varphi$ состоит в том, чтобы рассматривать ее как давление поляризационного типа, поскольку величина $\varphi$ возникает в пустом пространстве под действием одной лишь гравитации. Заметим, что давление такого же происхождения (обусловленное "истинной поляризацией вакуума") рассматривалось в [4] в связи с попыткой построения так называемой "инфляционной" модели "ранней" Вселенной. Наличие поляризационного давления $\varphi$ и плотности потока энергии $\Phi_{\alpha}$, по-видимому, может иметь принципиальное значение в областях вблизи сингулярностей.

Уравнения, получаемые от варьирования компонент метрики, содержащихся в ее бесследовой пространственной части, дают динамические уравнения для этих пяти компонент. Уравнение от $\left(u^{\alpha} u^{\beta} \delta g_{\alpha \beta}\right)$-варьирования является уравнением для компоненты $u^{\alpha} u^{\beta} g_{\alpha \beta}$. Это уравнение, по-сушеству, есть связь для данных Коши. Как и в обычном варианте, связь вводится в 4-объем, если она выполнена на начальной поверхности. Таким образом, в нашем подходе из четырех связей для данных Коши $\left(G_{\alpha}^{0}=0\right)$ остается только одна $\left(G_{0}^{0}=0\right)$. Остальные три преврашаются в условия для определения пространственных компонент вектора плотности потока энергии. Следовая часть пространственной части уравнений является уравнением для вычисления давления. Величины $\varphi$ и $\Phi_{\alpha}$ описывают реакцию материи, обеспечиваюшей постоянство системы отсчета в процессе варьирования.

Представляет интерес сравнить развитую нами схему с релятивистской теорией гравитации [5].

Автор благодарит Ю.А. Романова за критические замечания по поводу появления привилегированной системы отсчета, связанной с полем $u^{\alpha}(x)$. Работа выполнена при частичной поддержке Международного научно-технического центра (проект \#208).

\section{Список литературы}

[1] Л. Д. Ландау, Е. М. Лифииц. Теория поля. Изд. 7-е. М.: Наука, 1988.

[2] Ю. И. Неймарк, Н. А. Фуфаев. Динамика неголономных систем. М.: Наука, 1967.

[3] Ю. С. Владимиров. Системы отсчета в теории гравитации. М.: Энергоатомиздат, 1982.

[4] Я. Б. Зельдович. УФН. 1981. Т. 133. Вып. 3. С. 479.

[5] А.А. Логунов, М.А. Мествиришвили. Релятивистская теория гравитации. М.: Наука, 1989. 